\title{
ANÁLISE DA COMPOSIÇÃO CORPORAL E PRÁTICA DE ATIVIDADE FÍSICA EM MULHERES PÓS-MENOPAUSA
}

\author{
Eloise Werle Almeida, Universidade Estadual de Londrina - UEL, Londrina, Paraná- \\ Brasil \\ Marcia Greguol, Universidade Estadual de Londrina - UEL, Londrina, Paraná - Brasil
}

\begin{abstract}
RESUMO
O objetivo do estudo foi avaliar a concentração de gordura corporal e o nível de atividade física de mulheres pós-menopausa. Participaram da amostra 20 mulheres pós-menopausa com idade média de 54,6 $( \pm 3,05)$ anos. Foi aplicado o IPAQ para avaliar o nível de prática de atividade física e um questionário de percepções corporais após a menopausa. Foi feita uma análise com bioimpedância Biodynamics modelo 310, para avaliar o percentual de gordura e medida a circunferência abdominal, peso e estatura das participantes. $\mathrm{O}$ tempo médio de menopausa foi de $67( \pm 47,16)$ meses. Mulheres ativas tiveram melhores resultados de IMC, Circunferência Abdominal e Percentual de gordura corporal. 80\% das participantes foram classificadas como suficientemente ativas. Assume-se então que a prática de atividade física e um estilo de vida saudável são cruciais para a manutenção da saúde, e a prevenção de doenças advindas do envelhecimento em mulheres pósmenopausa.
\end{abstract}

Palavras-Chave: Menopausa; Composição corporal; Atividade física.

\section{BODY COMPOSITION AND PHYSICAL ACTIVITY ANALISYS IN POSTMENOPAUSAL WOMEN}

\begin{abstract}
The aim of this study was to evaluate body fat concentration and physical activity in postmenopausal women. The sample included by 20 postmenopausal women in mean age of 54,6 $\pm 3,05$ years. The IPAQ was applied to evaluate physical activity level. A postmenopausal body perception quastionnaire was also applied. An analysis with bioimpedance Biodynamics model 310 was performed to evaluate the body fat percentage. The participants' waist circunference, body mass and height were measured. Results showed a mean menopause duration of $67( \pm 47,16)$ months. Active women had better results of BMI, Waist Circunference and body fat percentage. $80 \%$ of participants were rate as sufficiently active. Based on this data, it is conclued that a lot of attention is needed in this population's lifestyle. Those results corroborate with the idea that physical activity and healthy lifestyle are crucial to health maintenance, and aging-related desease prevention in postmenopausal women.
\end{abstract}

Key-Words: Menopause; Body composition; Physical activity 


\section{ANÁLISIS DE LA COMPOSICIÓN CORPORAL Y PRÁCTICA DE LA ACTIVIDAD FÍSICA EN MUJERES POSMENOPÁUSICAS}

\section{RESUMEN}

El objetivo del estudio fue evaluar la concentración de la grasa y el nivel de actividad física corporal de las mujeres posmenopáusicas. Una muestra de 20 mujeres posmenopáusicas con una edad media de 54,6 $( \pm 3,05)$ años. IPAQ se utilizó para evaluar el nivel de actividad física y un cuestionario sobre la percepción del cuerpo después de la menopausia. Se realizó un análisis de bioimpedancia Biodinámica Modelo 310, para determinar el porcentaje de grasa corporal y la medida de la circunferencia de la cintura, peso Y altura de los participantes. La duración media de la menopausia fue de $67( \pm 47.16)$ meses. Las mujeres activas tenían mejores resultados que el IMC, circunferencia de cintura y porcentaje de grasa corporal. $80 \%$ de los participantes fueron clasificados como suficientemente activos. Se supone entonces que la práctica de la actividad física y estilo de vida saludable son cruciales para el mantenimiento de la salud y la prevención de las enfermedades relacionadas con la edad en las mujeres posmenopáusicas.

Palabras-Clave: La menopausia; La composición corporal; La actividad física. 


\section{INTRODUÇÃO}

A prevalência de obesidade vem aumentando de forma alarmante tanto em países desenvolvidos quanto em países em desenvolvimento. ${ }^{1}$ Definida de uma maneira simplificada, a obesidade é o acúmulo excessivo de gordura corporal. ${ }^{2}$ Dados do Ministério da Saúde (2012) colocam que 48,5\% dos brasileiros apresentam excesso de peso. E quando se trata de obesidade, $15,8 \%$ da população está nesta condição. A prevalência ainda se acentua com a idade, sendo que os maiores índices de obesidade são encontrados entre os 45 e 54 anos ( $37 \%$ entre homens e $55 \%$ entre mulheres). ${ }^{2}$

Francischi et al. $^{3}$ destacam que o envelhecimento está associado ao ganho de peso corporal, por fatores como o declínio da Taxa Metabólica Basal (quantidade de energia necessária para a manutenção das funções vitais do organismo) ${ }^{4}$ em consequência da perda de massa muscular, diminuição na prática de atividades físicas e aumento no consumo alimentar. Em mulheres, a partir dos 40 anos, esse quadro pode ser reforçado, já que nesse período, denominado climatério, ocorrem uma série de alterações hormonais que resultam no ganho de tecido adiposo e alteram a distribuição de gordura corporal, dentre outros sintomas. $^{5-8}$

O climatério é um fenômeno natural e decorrente da falência ovariana, cujas consequências provocam alterações endócrinas e metabólicas. ${ }^{9}$ Ele consiste no período de transição entre a vida reprodutiva e não reprodutiva da mulher. ${ }^{8,10}$ Acontece geralmente entre os 35 e 65 anos, e as alterações hormonais características desta fase provocam mudanças no ciclo menstrual. ${ }^{10}$ Essas graduais mudanças caminham para o último episódio de sangramento uterino, a menopausa. ${ }^{11}$

As alterações hormonais durante o climatério e a menopausa são o restante de poucos folículos ovarianos e, além disso, os folículos remanescentes se tornam menos sensíveis à ação dos hormônios LH (Hormônio Luteinizante) e FSH (Hormônio Folículo Estimulante), que estimulam os ovários a produzir estrógeno e progesterona, ${ }^{12}$ mesmo que a taxa desses hormônios permaneça elevada. Como a resposta dos ovários a esses hormônios se torna menor, é também menor o número de folículos que amadurecem. ${ }^{11,13}$

Após a menopausa, a massa óssea e a massa magra tendem a diminuir e a massa gordurosa tende a aumentar. ${ }^{11}$ É sugerido que a massa gorda sofra um aumento de 5 a $10 \%$ e a massa 
magra diminua 2,5\% por década. Mulheres na pós-menopausa podem ter $20 \%$ a mais de massa gordurosa do que mulheres pré-menopausa. ${ }^{7}$

Os esteróides ovarianos atuam alterando o metabolismo do tecido adiposo ${ }^{11}$. Com a diminuição dos níveis desses esteróides sexuais, há uma diminuição da atividade lipolítica no tecido adiposo abdominal e da ação da lípase lipoproteica no tecido adiposo fêmurogluteal. ${ }^{7,11}$ Esse fato pode influenciar o maior acúmulo de gordura abdominal (gordura andróide) e a diminuição do acúmulo de gordura nos quadris e na coxa (gordura ginecóide) na mulher climatérica e menopáusica. ${ }^{11}$

Para avaliar isoladamente a distribuição de gordura, utiliza-se a medida da Circunferência Abdominal. Ela fornece mais informações sobre o risco de doenças cardiovasculares gerado por esse acúmulo de adiposidade abdominal do que o Índice de Massa Corporal, que fornece resultados da obesidade global. ${ }^{14-15}$

A redução dos níveis dos esteróides ovarianos também se reflete no sistema ósseo de mulheres pós-menopausa. A partir deste período a atividade de formação de massa óssea tende a diminuir e se tornar menor que o processo de reabsorção, levando à diminuição da massa óssea. Quando este processo se torna mais intenso, pode resultar em um quadro de osteoporose, caracterizado pela deterioração da microarquitetura dos ossos e baixa massa óssea. $^{16}$

As alterações da composição corporal decorrentes da menopausa, acompanhadas com a diminuição da prática de atividade física, associada ao aumento da idade, podem influenciar a Taxa Metabólica de Repouso (taxa mínima de energia consumida para manter as funções fisiológicas no estado de repouso). Esta depende da quantidade de massa magra e de tecidos metabolicamente ativos como o coração, rins e fígado. ${ }^{14}$

Sendo assim, a redução de massa magra pode provocar a diminuição da Taxa Metabólica de Repouso e, a partir disto, pode haver ganho de peso e gordura corporal e aumento do risco de doenças crônico degenerativas. ${ }^{14}$

A literatura aponta também que a redução de massa magra e o incremento de gordura corporal podem influenciar na realização de atividades funcionais da vida diária. Foram encontrados resultados que apontam que o aumento da adiposidade corporal, seja ela 
global ou abdominal, exerce efeito negativo na capacidade funcional para realizar as atividades da vida diária em mulheres acima de 47 anos. $^{17}$

Estudos colocam que o acúmulo de gordura na região abdominal é um fator de risco considerável para o desenvolvimento de doenças cardiovasculares. ${ }^{1,5-7}$ Dessa maneira, é maior o risco desse tipo de patologia em mulheres climatéricas e pós-menopausa. Como fator agravante, com as doenças cardiovasculares e a obesidade associam-se também uma série de outras doenças, como diabetes tipo II e hipertensão arterial. ${ }^{3}$

Para o controle e prevenção dessas doenças, sabe-se que a atividade física atua de forma positiva. Quando praticada de maneira regular e sistemática, melhora a capacidade cardiovascular e respiratória do organismo, além de diminuir a pressão arterial em hipertensos. Também combatendo a obesidade, a prática da atividade física atua de maneira positiva na composição corporal, diminuindo o percentual de gordura e aumentando a massa magra. ${ }^{1,3,10}$

Sendo assim, um programa de atividade física pode trazer muitos benefícios para a mulher no climatério ou menopausa. ${ }^{1}$ A prática pode contribuir no controle da obesidade e minimizar as mudanças de distribuição de gordura corporal decorrentes das alterações hormonais desse período, ${ }^{3}$ e, consequentemente, prevenir o desenvolvimento das doenças crônico-degenerativas mais comuns.

A partir do acima exposto, encontra-se um problema para o estudo: Pode a atividade física atuar de maneira positiva na prevenção do aumento na porcentagem de gordura, especialmente na região abdominal, decorrente do climatério e menopausa em mulheres nesse período?

O presente estudo objetiva avaliar a concentração de gordura corporal e o nível de atividade física de mulheres no período pós-menopausa.

\section{MATERIAIS E MÉTODOS}

A população do estudo foi composta por mulheres londrinenses entre 50 e 60 anos de idade, que estivessem no período pós-menopausa. Assim, o estudo contou com uma amostra de 20 mulheres moradoras da cidade de Londrina, com idades entre os 50 e 60 anos, todas já com interrupção definitiva do seu ciclo menstrual. O estado menopausal foi 
definido com base na história menstrual do último ano, sendo considerada pós-menopausa quando havia amenorreia completa há pelo menos um ano. ${ }^{10}$

Foi feita a medida da circunferência abdominal, utilizando uma fita métrica maleável com um metro e meio de comprimento e medida em milímetros. Segundo recomendações da OMS, a medida foi realizada no maior perímetro abdominal entre a crista ilíaca e a última costela. Foi considerado um risco de complicações metabólicas aumentado perímetro maior que $88 \mathrm{~cm}^{18}$

Foram mensuradas ainda a massa corporal e a estatura. A partir destas medidas, foi calculado o IMC por meio da fórmula $I M C=\frac{\text { massa }}{\operatorname{sinatura} a^{2}}$. Foram considerados normais valores de IMC entre 18,5 e 24,99 $\mathrm{Kg} / \mathrm{m}^{2}$, sobrepeso IMC entre 25 e 29,99 $\mathrm{Kg} / \mathrm{m}^{2}$, obesidade grau I valores entre $30 \mathrm{Kg} / \mathrm{m}^{2}$ e $34,99 \mathrm{Kg} / \mathrm{m}^{2}$, obesidade grau II valores entre 35 $\mathrm{Kg} / \mathrm{m}^{2}$ e $39,99 \mathrm{Kg} / \mathrm{m}^{2}$ e obesidade grau III Valores de IMC maior que $40 \mathrm{Kg} / \mathrm{m}^{2}{ }^{18}$

Foi aplicado um questionário de percepções de mudanças corporais antes e depois da menopausa, criado pela pesquisadora. Ele teve como objetivo avaliar se as participantes da pesquisa notaram mudanças importantes na distribuição de gordura corporal durante o climatério e depois da menopausa e também quais foram os sintomas mais marcantes deste período. Para isso, a participante respondeu perguntas sobre a última menstruação, as alterações corporais que ela notou após esse acontecimento, os sintomas que precederam a menopausa e se houve prática de atividade física durante o climatério.

Para avaliar o nível atual de prática de atividade física das participantes foi aplicada a versão curta do Questionário Internacional de Atividade Física (IPAQ) ${ }^{20}$ Nele as perguntas foram relacionadas ao tempo gasto com a prática de atividade física nos sete dias precedentes. As participantes foram classificadas em insuficientemente ativas ou suficientemente ativas, de acordo com a quantidade e intensidade de prática de atividade física durante a semana que precedeu a pesquisa.

Para avaliar o percentual de gordura corporal foi utilizado um aparelho de bioimpedância tetrapolar da marca Biodynamics, modelo 310, Estados Unidos. 


\section{Procedimentos}

Mulheres potencialmente elegíveis para a amostra foram convidadas a participar do estudo (amostra por conveniência). Estas foram abordadas em centros estéticos ou clínicas médicas. A proposta e os procedimentos do estudo foram expostos às participantes. Caso elas atendessem o critério para participação na pesquisa (estágio pós-menopausa) e aceitassem participar, era assinado o Termo de Consentimento Livre e Esclarecido para participação. Feito isso foi agendado um horário para fazer as avaliações e os questionários.

No dia agendado foram aplicados os questionários relativos à atividade física e às percepções de mudanças corporais. Não foi limitado tempo para o preenchimento dos questionários. Também nesta ocasião foi mensurada a circunferência abdominal, massa corporal e estatura.

A avaliação do percentual de gordura com a bioimpedância também foi realizada neste dia. Foi solicitado às participantes, que já estavam instruídas dos procedimentos (jejum de pelo menos 4 horas), que retirassem qualquer adereço de metal que estivessem utilizando. Foram colados dois eletrodos na mão direita (punho e dorso da mão) e dois eletrodos no pé direito (tornozelo e dorso do pé). Os dados da participante (sexo, idade, peso e estatura), solicitados pelo aparelho, foram digitados na máquina e a análise foi feita pela bioimpedância. Os resultados de percentual de gordura foram impressos imediatamente após o teste pela máquina.

As avaliações foram feitas individualmente com as participantes, com apenas um pesquisador realizando a coleta. Concluída essa etapa os dados foram informatizados e analisados.

\section{Análise Estatística}

Os dados foram analisados inicialmente por meio de estatística descritiva, com apresentação dos valores médios e desvios padrão. Foi aplicado o teste "t" de student para amostras independentes para verificar diferenças entre os grupos. Em seguida, a fim de se verificar a possível associação entre os níveis de atividade física e os indicadores de gordura corporal, foi realizado teste de correlação de Pearson, adotando-se nível de significância de 5\%. As variáveis categóricas foram apresentadas em valores percentuais. Todas as análises foram realizadas no programa estatístico SPSS versão 17.0. 


\section{RESULTADOS}

A análise descritiva dos dados está descrita na Tabela 1. Quanto à prática de atividade física avaliada pelo Questionário IPAQ (Questionário Internacional de Atividade Física Versão Curta), $80 \%$ das participantes foram classificadas como suficientemente ativas (prática de 150 minutos ou mais de atividades físicas semanais). Apenas 20\% das mulheres da amostra não praticavam a marca de 150 minutos de atividades físicas em uma semana e foram classificadas como insuficientemente ativas.

Tabela 1- Análise descritiva dos dados da amostra

\begin{tabular}{lcccc}
\hline & Mínimo & Máximo & Média & $\begin{array}{c}\text { Desvio } \\
\text { Padrão }\end{array}$ \\
\hline Idade (anos) & 50 & 60 & 54,60 & 3,05 \\
Tempo Menopausa (meses) & 12 & 144 & 67,00 & 47,16 \\
IMC $\left(\mathbf{K g} / \mathbf{m}^{\mathbf{2}}\right)$ & 20,70 & 38,80 & 27,01 & 4,70 \\
CA $(\mathbf{c m})$ & 69,80 & 114,00 & 86,13 & 12,61 \\
Gordura (\%) & 21,30 & 43,00 & 31,81 & 5,73 \\
\hline
\end{tabular}

Ao se aplicar o teste " $t$ " de student para amostras independentes para verificar diferenças entre mulheres suficientemente e insuficientemente ativas, notaram-se diferenças significativas nas variáveis IMC $(p=0,004)$, circunferência abdominal $(p=0,045)$ e percentual de gordura $(\mathrm{p}=0,025)$, todas em favor do grupo ativo. As médias e desvios destas variáveis por grupo estão apresentadas na Tabela 2.

Tabela 2 - Resultados do teste t-independente para verificar diferenças entre mulheres suficientemente e insuficientemente ativas.

\begin{tabular}{llrrr}
\hline & Atividade & & \multicolumn{2}{c}{ Desvio } \\
& Física & N & Média & \multicolumn{1}{c}{ Padrão } \\
\hline IMC & Ativas & 16 & 25,61 & 3,62 \\
& Sedentárias & 4 & 32,63 & 4,71 \\
CA & Ativas & 16 & 82,35 & 9,78 \\
& Sedentárias & 4 & 101,25 & 12,18 \\
Gordura & Ativas & 15 & 30,33 & 5,11 \\
& Sedentárias & 4 & 37,35 & 4,83 \\
\hline
\end{tabular}

Foi feito o teste de correlação de Pearson a fim de se verificar relações entre as variáveis e os resultados constam na Tabela 3. 
Tabela 3 - Resultados do Teste de Correlação de Pearson entre as variáveis

\begin{tabular}{|c|c|c|c|c|c|c|}
\hline & \multicolumn{6}{|c|}{ Tempo } \\
\hline & & Idade & Menop & IMC & $\mathbf{C A}$ & Gordura \\
\hline \multirow[t]{2}{*}{ Idade } & $\begin{array}{l}\text { Valor da } \\
\text { correlação }\end{array}$ & 1 &, $524^{*}$ &, 037 &, 045 &, 053 \\
\hline & Significância (p) & & ,018 & 877 & ,852 & ,829 \\
\hline \multirow[t]{2}{*}{$\begin{array}{l}\text { Tempo de } \\
\text { menopausa }\end{array}$} & $\begin{array}{l}\text { Valor da } \\
\text { correlação }\end{array}$ &, $524^{*}$ & 1 &, 175 &, 061 &, 150 \\
\hline & Significância (p) & ,018 & & ,462 & ,798 &, 540 \\
\hline \multirow[t]{2}{*}{ IMC } & $\begin{array}{l}\text { Valor da } \\
\text { correlação }\end{array}$ & ,037 &, 175 & 1 &, $891^{* *}$ & $902^{* *}$ \\
\hline & Significância (p) & 877 & ,462 & &, 000 &, 000 \\
\hline \multirow[t]{2}{*}{ CA } & $\begin{array}{l}\text { Valor da } \\
\text { correlação }\end{array}$ & ,045 & ,061 &, $891^{* *}$ & 1 &, $781^{* *}$ \\
\hline & Significância (p) &, 852 & ,798 &, 000 & &, 000 \\
\hline \multirow[t]{2}{*}{ Gordura } & $\begin{array}{l}\text { Valor da } \\
\text { correlação }\end{array}$ &, 053 &, 150 &, $902^{* *}$ &, $781^{* *}$ & 1 \\
\hline & Significância (p) & ,829 & ,540 &, 000 &, 000 & \\
\hline
\end{tabular}

Os resultados mostram que ocorreram correlações fortes entre tempo de menopausa e idade (quanto maior a idade maior o tempo de menopausa), entre circunferência abdominal e IMC (quanto maior o IMC maior a CA), entre o IMC e o percentual de gordura (quanto maior o IMC maior o percentual de gordura corporal) e entre o percentual de gordura e a $\mathrm{CA}$ (quanto maior o percentual de gordura, maior a CA).

Para que as mulheres fossem classificadas dentro de padrões considerados saudáveis, elas foram divididas nos seguintes grupos:

- Quanto ao IMC: Grupo 1 (IMC menor do que 25 - eutrofia); Grupo 2 (IMC de 25 a 29,9 - sobrepeso); Grupo 3 (IMC maior ou igual a 30 - obesidade);

- Quanto à circunferência abdominal: Grupo 1 (CA até $80 \mathrm{~cm}$ - ideal); Grupo 2 (CA de 80,1 a $88 \mathrm{~cm}$ - aceitável); Grupo 3 (CA acima de $88 \mathrm{~cm}$ - risco cardiovascular);

- Quanto ao percentual de gordura: Grupo 1 (percentual de gordura até $27 \%$ desejável); Grupo 2 (percentual de gordura acima de $27 \%$ - acima do desejável). ${ }^{21}$

Os resultados das porcentagens foram os seguintes: 
Tabela 4 - Classificação das participantes pelo índice de massa corporal

\begin{tabular}{rrrc}
\hline & & Frequência & Porcentagem \\
\hline Grupos & 1 & 8 & $40 \%$ \\
& 2 & 8 & $40 \%$ \\
& 3 & 4 & $20 \%$ \\
\hline
\end{tabular}

Tabela 5 - Classificação das participantes pela circunferência abdominal

\begin{tabular}{rrrr}
\hline & & Frequência & Porcentagem \\
\hline Grupos & 1 & 8 & $40 \%$ \\
& 2 & 3 & $15 \%$ \\
& 3 & 9 & $45 \%$ \\
\hline
\end{tabular}

Tabela 6 - Classificação das participantes pelo percentual de gordura

\begin{tabular}{lrrr}
\hline & & Frequência $^{\mathbf{1}}$ & Porcentagem $^{-}$ \\
\hline Grupos & 1 & 3 & $15,8 \%$ \\
& 2 & 16 & $84,2 \%$ \\
\hline
\end{tabular}

Em relação ao questionário de percepções corporais antes e depois da menopausa, os resultados demonstram que 55\% das participantes praticaram atividades físicas durante o climatério. Também 55\% das mulheres apontaram que perceberam um maior acúmulo de gordura abdominal depois da menopausa. Os sintomas do climatério mais citados dentre as participantes foram: ondas de calor e fogachos (70\%), insônia (25\%) e sintomas emocionais e psicológicos $(15 \%)$.

\section{DISCUSSÃO}

As alterações hormonais decorrentes da menopausa podem provocar uma série de mudanças corporais na mulher. Dentre elas, destaca-se o ganho de gordura abdominal. A redução dos níveis de esteróides sexuais femininos provoca uma menor atividade da enzima lipase na região fêmuro-gluteal e consequentemente o menor acúmulo de tecido adiposo neste local. Desta forma o padrão de distribuição de gordura corporal se altera de ginecóide para andróide, no qual o maior depósito de adipócitos se dá na região do abdômen. ${ }^{9}$

Esta característica pôde ser ratificada no presente estudo. O grupo analisado apresentou IMC médio de $27,01 \pm 4,70 \mathrm{Kg} / \mathrm{m}^{2}$ e o valor da média da circunferência abdominal foi de

\footnotetext{
${ }^{1}$ Neste caso foram contabilizadas apenas 19 participantes devido à impossibilidade de realização da avaliação de percentual de gordura por parte de uma participante.

Conexões: revista da Faculdade de Educação Física da UNICAMP, Campinas, v. 11, n. 3, p. 129-146, jul./set. 2013. 
$86,13 \mathrm{~cm} \pm 12,61 \mathrm{~cm}$. É possível então caracterizar o grupo em uma taxa de sobrepeso em relação ao primeiro resultado e, analisando a obesidade abdominal, coloca-se o grupo com uma média acima da ideal para esta variável.

Mulheres pós-menopausa moradoras da cidade de São Paulo obtiveram valores médios de IMC de $28 \mathrm{Kg} / \mathrm{m}^{2}$ sendo que $65,5 \%$ estavam dentro do padrão eutrófico. ${ }^{22}$ Já no presente estudo $40 \%$ da amostra indicava eutrofia. As demais participantes caracterizaram-se com sobrepeso (40\%) ou obesas (20\%). Em relação aos valores de circunferência abdominal, as participantes deste estudo obtiveram melhores resultados quando comparadas a uma população semelhante da cidade de Botucatu, que tiveram valor médio de circunferência abdominal de $91,4 \mathrm{~cm} .^{23}$

A sarcopenia (perda de massa muscular) é uma das mudanças advindas do envelhecimento. $^{23}$ Este fator, somado à redução de massa óssea, consequência da diminuição dos níveis de estrógeno que provoca alterações nas atividades de formação e reabsorção óssea, ${ }^{16}$ pode se refletir no percentual de gordura corporal. As participantes deste estudo apresentaram, em média, $31,81 \% \pm 5,73 \%$ de gordura corporal. Ainda entre as mulheres pesquisadas, $84,2 \%$ estavam acima dos $27 \%$ de gordura corporal propostos por Pollock e Wilmore $(1993)^{21}$ como um valor desejável para a promoção e manutenção da saúde para esta faixa etária. No entanto, estes índices se demonstram positivos em relação a uma pesquisa de Brasília, na qual o percentual médio de gordura encontrado em mulheres pós menopausa foi de $40 \%{ }^{24}$

Com a prática de atividade física, é possível controlar a obesidade e amenizar as mudanças de distribuição de gordura corporal decorrentes das alterações hormonais da menopausa. ${ }^{3}$ No presente estudo, a prevalência de mulheres suficientemente ativas, que realizavam pelo menos 150 minutos de atividade físicas semanais, foi de $80 \%$. Estes valores se mostram positivos quando comparados a mulheres pós-menopausa paulistanas, entre as quais apenas $47,8 \%$ foram consideradas ativas ou muito ativas. ${ }^{22}$ Os resultados desta variável no presente estudo também são superiores aos observados no Brasil em 2003 com mulheres de 40 aos 65 anos avaliadas pelo questionário IPAQ em um estudo epidemiológico, no qual apenas $62 \%$ foram classificadas como ativas. ${ }^{25}$

Ao comparar as participantes ativas e não ativas do estudo, foram verificadas diferenças significativas nas variáveis IMC, circunferência abdominal e percentual de gordura. As 
mulheres ativas tiveram percentual de gordura, IMC e circunferência abdominal menores do que aquelas insuficientemente ativas. Estes dados corroboram a ideia de que a prática de atividades físicas pode ser efetiva para a saúde de adultas e idosas. ${ }^{23}$ A prática regular e sistemática de atividade física, como o treinamento com pesos, por exemplo, pode promover o ganho de massa muscular. Isto pode influenciar diretamente na redução do percentual de gordura corporal. Também é possível aumentar o gasto energético de repouso e a demanda metabólica com o ganho de massa muscular gerado pela atividade física. Estes benefícios previnem e combatem o ganho de peso e gordura corporal, e se refletem em menores taxas de IMC e circunferência abdominal. ${ }^{23}$

A literatura também aponta que o treinamento resistido pode ter um papel importante na prevenção da perda de massa óssea e na manutenção da Densidade Mineral Óssea. No caso da deficiência de estrogênio encontrada em mulheres pós-menopausa, o sistema ósseo tenta manter ou aumentar a massa óssea através dos estímulos ósseos gerados pelas contrações musculares durante o exercício. ${ }^{26}$ Um maior percentual de massa óssea pode se refletir em um menor percentual de gordura corporal.

Além da prática de atividades sistematizadas, as práticas domésticas ou de lazer estão englobadas no instrumento utilizado para avaliar o nível de atividade física das participantes (IPAQ). Estudos apontam que estas atividades podem estar associadas a efeitos favoráveis no nível psicológico, social e na qualidade de vida. No entanto, parecem ser necessários sessenta minutos de atividades físicas de maior intensidade por dia para efeitos mais significativos no aspecto físico. ${ }^{25}$ Este fato pode explicar o maior percentual de mulheres com sobrepeso ( $40 \%$ com IMC entre 25 e $29,9 \mathrm{Kg} / \mathrm{m}^{2}$ ) e com percentual de gordura acima do desejável (84,2\% acima dos $27 \%$ de gordura desejáveis), mesmo entre aquelas participantes suficientemente ativas.

A concentração excessiva de gordura na região abdominal está relacionada com o desenvolvimento de diversas disfunções metabólicas e também doenças cardiovasculares. $^{27-28}$ Adicionalmente à menopausa, os maus hábitos alimentares e de vida contribuem para o surgimento destas doenças crônico-degenerativas, que nos últimos anos têm sido causa de mortalidade em mulheres mais que câncer de mama e AIDS. ${ }^{29}$ Outro risco do acúmulo excessivo de adiposidade abdominal é o aumento da pressão arterial. Estima-se que em mulheres adultas, um aumento de $2,5 \mathrm{~cm}$ na circunferência abdominal corresponda a um aumento na pressão arterial sistólica de $1 \mathrm{~mm} \mathrm{Hg.}{ }^{27} \mathrm{O}$ desenvolvimento Conexões: revista da Faculdade de Educação Física da UNICAMP, Campinas, v. 11, n. 3, p. 129-146, jul./set. 2013. 
da aptidão cardiorrespiratória por meio da prática de atividades físicas, juntamente com a mudança do estilo de vida, pode prevenir e diminuir o risco de um quadro hipertensivo e de doença cardiovascular em mulheres adultas e idosas. ${ }^{27,30}$

Esta relação entre atividade física e menor adiposidade abdominal pode ser observada no presente estudo. Quando comparados os valores de circunferência abdominal de mulheres ativas e insuficientemente ativas, o primeiro grupo apresentou média de $82,35 \mathrm{~cm} \pm 9,78$ cm enquanto o grupo de mulheres não ativas obteve média de $101,25 \mathrm{~cm} \pm 4,83 \mathrm{~cm}$.

Ainda foi possível observar que quanto maiores os valores do Índice de Massa Corporal das participantes, também eram maiores os valores de Circunferência Abdominal e percentual de gordura corporal. Estes resultados podem indicar que indivíduos com excesso de peso podem desenvolver outras condições de saúde associadas. ${ }^{3}$

Estes resultados podem indicar que realmente as alterações hormonais decorrentes da menopausa exercem grande influência na distribuição de gordura corporal. A diminuição dos níveis de esteróides sexuais promove o maior acúmulo de adipócitos na região abdominal, caracterizando o padrão andróide. Também a baixa significativa dos hormônios sexuais pode provocar sintomas desconfortáveis, com destaque para as ondas de calor, insônia, depressão e ansiedade. ${ }^{10}$ De acordo com os resultados apresentados no questionário de percepções corporais antes e depois da menopausa, as mulheres apresentaram de fato estes sintomas, sendo as ondas de calor a queixa mais frequente.

Além das ondas de calor, também sintomas de ordem emocional e psicológica foram colocados pelas participantes, dentre eles depressão e ansiedade, manifestadas por $15 \%$ das mulheres. Estes valores se mostram inferiores quando comparados ao estudo de Pereira et al., ${ }^{10}$ que avaliou a ansiedade em mulheres no climatério e pós-menopausa. Neste caso, a prevalência de ansiedade foi de 49,8\%. Este autor coloca que além das causas hormonais para o surgimento de desordens emocionais, também as turbulências emocionais decorrentes da transição de um período fértil para a menopausa podem gerar ansiedade e outros sintomas psicológicos.

Levando em consideração as argumentações apresentadas, é possível inferir que de fato o período da menopausa interfere no perfil e na composição corporal das mulheres. Existe nesta fase um maior acúmulo de gordura na região abdominal, aumentando-se o risco de desenvolvimento de doenças crônico-degenerativas comuns ao envelhecimento. No Conexões: revista da Faculdade de Educação Física da UNICAMP, Campinas, v. 11, n. 3, p. 129-146, jul./set. 2013. 
entanto, é plausível admitir que a prática de atividades físicas, sejam elas atividades domésticas ou exercícios físicos sistematizados, pode minimizar estas consequências da menopausa.

Mulheres ativas, que realizam pelo menos 150 minutos de atividades físicas semanais, apresentam um perfil lipídico menos favorável ao surgimento de doenças cardiovasculares, hipertensão arterial e diabetes mellitus. A menor quantidade de gordura corporal pode até mesmo ser um fator preventivo ao surgimento de tumores na mama ${ }^{31}$. Sendo assim, é possível considerar a atividade física como fundamental para a manutenção da saúde da mulher pós-menopáusica.

Uma possível limitação do presente estudo é o fato de a amostra ter sido selecionada por conveniência, sendo boa parte das mulheres participantes convidadas em centros de estética. Esta situação pode ter contribuído para o elevado número de mulheres fisicamente ativas identificadas na amostra. Além disso, não foram controlados a dieta nem tampouco o consumo de medicamentos pelas participantes, fatores estes que podem exercer influências nas variáveis da composição corporal. Apesar disso, os dados aqui levantados reforçam a importância da manutenção de um estilo de vida fisicamente ativo por mulheres antes e após a menopausa, como forma de amenizar os desconfortos físicos e psicológicos advindos neste período.

\section{CONCLUSÃO}

O objetivo do presente estudo era avaliar a concentração de gordura corporal e o nível de atividade física de mulheres no período pós-menopausa. Neste sentido, foi possível observar como o percentual de gordura corporal, a distribuição de gordura corporal e a prática de atividades físicas são fatores importantes na saúde da mulher pós-menopausa.

As mulheres pós-menopausa classificadas como insuficientemente ativas, que não praticavam pelo menos 150 minutos de atividades físicas semanais, tiveram resultados de IMC, percentual de gordura corporal e circunferência abdominal mais preocupantes que as mulheres ativas. Estes resultados confirmam a hipótese de que a prática de atividades físicas tem papel fundamental na manutenção do peso corporal, aumento de massa muscular e, consequentemente, pode prevenir o desenvolvimento de doenças crônico degenerativas, como hipertensão e doenças cardiovasculares. 
No entanto, $60 \%$ das participantes da pesquisa estavam com sobrepeso ou obesidade, quando analisado o IMC, $45 \%$ estavam com a medida da circunferência abdominal acima do aceitável e 84,2\% estavam com um percentual de gordura acima do desejável, incluindo mulheres ativas e sedentárias. Estes resultados podem indicar a forte influência da diminuição dos níveis de esteróides sexuais nos padrões corporais da mulher pósmenopausa.

Com base nestes dados, conclui-se que é necessária muita atenção no estilo de vida desta população. A prática de atividade física e um estilo de vida saudável são cruciais para a manutenção da saúde e a prevenção de doenças advindas do envelhecimento em mulheres que passaram pela menopausa.

\section{REFERÊNCIAS}

${ }^{1}$ MONTEIRO, R. C. A.; RIETHER, P. T. A.; BURINI, R. C. Efeito de um programa misto de intervenção nutricional e exercício físico sobre a composição corporal e os hábitos alimentares de mulheres obesas em climatério. Revista de Nutrição, Campinas, v. 17, n. 4, p. 479-489, out./dez. 2004.

${ }^{2}$ PINHEIRO, A. R. O.; FREITAS, S. F. T.; CORSO, A. C. T. Uma abordagem epidemiológica da obesidade. Revista de Nutrição, Campinas, v. 17, n. 4, p. 523-533, out./dez. 2004.

${ }^{3}$ FRANCISCHI, R. P. P. et al. Obesidade: atualização sobre sua etiologia, morbidade e tratamento. Revista de Nutrição, v. 13, n. 1, p.17-28, jan./abr. 2000.

${ }^{4}$ WAHRLICH, V.; ANJOS, L. A. Aspectos históricos e metodológicos da medição e estimativa da taxa metabólica basal: uma revisão de literatura. Caderno de Saúde Pública, Rio de Janeiro, v. 17, n. 4, p. 801-817, jul./ago. 2001.

${ }^{5}$ REIS, C. M. R. F. et al. Composição corpórea, distribuição de gordura e metabolismo de repouso em mulheres histerectomizadas no climatério: há diferenças de acordo com a forma da administração de estrogênio? Arquivos Brasileiros de Endocrinologia e Metabologia, São Paulo, v. 44, n. 2, abr. 2000.

Conexões: revista da Faculdade de Educação Física da UNICAMP, Campinas, v. 11, n. 3, p. 129-146, jul./set. 2013. 
${ }^{6}$ RASKIN, D. B. F. et al. Fatores associados à obesidade e ao padrão andróide de distribuição da gordura corporal em mulheres climatéricas. Revista Brasileira de Ginecologia e Obstetrícia, Rio de Janeiro, v. 22, n. 7, p. 435-441, 2000.

${ }^{7}$ DE LORENZI, D. R. S. et al. Prevalência de sobrepeso e obesidade no climatério. Revista Brasileira de Ginecologia e Obstetrícia, Rio de Janeiro, v. 27, n. 8, p. 479-484, 2005.

${ }^{8}$ SCHMITT, A. C. B.; CARDOSO M. R. A.; ALDRIGHI J. M. Tendências da mortalidade em mulheres brasileiras no climatério. Revista Brasileira de Crescimento e Desenvolvimento Humano, São Paulo, v. 18, n.1, p.11-15, 2008.

${ }^{9}$ FERNANDES, L. F. C.; ALDRIDHI, A. P. S.; ALDRIGHI, J. M. Gordura abdominal visceral após a menopausa: novo tratamento? Revista da Associação Médica Brasileira, São Paulo, v. 51, n. 3, p.121-132, 2005.

${ }^{10}$ PEREIRA, W. M. P. et al. Ansiedade no climatério: prevalência e fatores associados. Revista Brasileira de Crescimento e Desenvolvimento Humano, São Paulo, v. 19, n.1, p. 89-97, 2009.

${ }^{11}$ PINOTTI, J. A.; HALBE, H. W.; HEGG, R. Menopausa. São Paulo: Roca, 1995.

12 PARDINI, D. P. Alterações hormonais na mulher atleta. Arquivos Brasileiros de Endocrinologia e Metabologia, São Paulo, v. 45, n. 4, p. 343-351, ago. 2001.

${ }^{13}$ TRIEN, S. F. Menopausa: a grande transformação. Rio de Janeiro: Rosa dos Tempos, 1991.

${ }^{14}$ BONGANHA, V. et al. Taxa metabólica de repouso e composição corporal em mulheres pós-menopausa. Arquivos Brasileiros de Endocrinologia e Metabologia, v. 56, n. 4, p. 755- 759, 2009. 
${ }^{15}$ ORSATTI, F. L. et al. Indicadores antropométricos e as doenças crônicas não transmissíveis em mulheres na pós-menopausa da região Sudoeste do Brasil. Revista Brasileira de Ginecologia e Obstetrícia, v. 30, n. 4, p. 182-189, 2008.

${ }^{16}$ NAVEGA, M. T.; OISHI, J. Comparação da qualidade de vida relacionada à saúde entre mulheres na pós-menopausa praticantes de atividade física com e sem osteoporose. Revista Brasileira de Reumatologia, São Paulo, v. 47, n. 4, p. 258-264, jul./ago. 2007.

${ }^{17}$ RASO, V. A adiposidade corporal e a idade prejudicam a capacidade funcional para realizar as atividades da vida diária de mulheres acima de 47 anos. Revista Brasileira de Medicina do Esporte, v. 8, n. 6, p. 225-234, nov./dez. 2002.

${ }^{18}$ ORGANIZAÇÃO MUNDIAL DA SAÚdE (OMS). Disponível em: < http://www.who.int/topics/en/>. Acesso em: 09 abr. 2012.

${ }^{19}$ PEIXOTO, M. R. G.; BENÍCIO, M. H. D.; JARDIM, P. C. B. V. Validade do peso e da altura auto-referidos: o estudo de Goiânia. Revista de Saúde Pública, São Paulo, v. 40, n. 6, p. 1065-1072, 2006.

${ }^{20}$ MATSUDO, S. et al. Questionário Internacional de Atividade Física (IPAQ): estudo de validade e reprodutibilidade no Brasil. Revista Atividade Física e Saúde, Londrina, v. 6, n. 2, p. 01-18, 2001.

${ }^{21}$ POLLOCK, M. L.; WILMORE, J. H. Exercícios na saúde e na doença: validação e prescrição para prevenção e reabilitação. Rio de Janeiro: Medsi, 1993. p. 233-362.

${ }^{22}$ FRANÇA, A. P.; ALDRIGHI, J. M.; MARUCCI, M. F. N. Fatores associados à obesidade global e à obesidade abdominal em mulheres pós-menopausa. Revista Brasileira de Saúde Materno Infantil, Recife, v. 8, n. 1, p. 65-73, jan./mar. 2008.

${ }^{23}$ TREVISAN, M. C.; BURINI, R. C. Metabolismo de repouso de mulheres pósmenopausa submetidas a programa de treinamento com pesos (hipertrofia). Revista Brasileira de Medicina do Esporte, São Paulo, v. 13, n. 2, p.133-137, mar./abr. 2007.

Conexões: revista da Faculdade de Educação Física da UNICAMP, Campinas, v. 11, n. 3, p. 129-146, jul./set. 2013. ISSN: 1983-9030 
${ }^{24}$ CHAVES, L. M. et al. Relação entre variáveis da composição corporal e densidade mineral óssea em mulheres idosas. Revista Brasileira de Medicina do Esporte, São Paulo, v. 11, n. 6, p. 352- 356, nov./dez. 2005.

${ }^{25}$ GUIMARÃES, A. C. A.; BAPTISTA, F. Atividade física habitual e qualidade de vida de mulheres na meia-idade. Revista Brasileira de Medicina do Esporte, São Paulo, v. 17, n. 5, p. 305- 309, set./out. 2011.

${ }^{26}$ KEMPER, C. et al. Efeitos da natação e do treinamento resistido na densidade mineral óssea de mulheres idosas. Revista Brasileira de Medicina do Esporte, São Paulo, v.15, n.1, p. 10-13, jan./fev. 2009.

${ }^{27}$ KRAUSE, M. P. et al. Associação de aptidão cardiorrespiratória e circunferência abdominal com hipertensão em mulheres idosas brasileiras. Arquivos Brasileiros de Cardiologia, São Paulo, v. 93, n.1, p. 2-8, 2009.

${ }^{28}$ ALMEIDA, R. T.; ALMEIDA, M. M. G.; ARAÚJO, T. M. Obesidade abdominal e risco cardiovascular: desempenho de indicadores antropométricos em mulheres. Arquivos Brasileiros de Cardiologia, São Paulo, v. 92, n. 5, 2009.

${ }^{29}$ MEDEIROS, S. L.; PADIAL, R. Doença arterial coronária no climatério e exclusão social. Saúde e Sociedade, São Paulo, v. 16, n.1, p. 45-56, jan./ abr. 2007.

${ }^{30}$ SILVA, R. B. et al. Atividade física habitual e risco cardiovascular na pós-menopausa. Revista da Associação Médica Brasileira, São Paulo, v. 52, n. 4, p. 242-246, 2006.

${ }^{31}$ CIBEIRA, G. H.; GUARAGNA, R. M. Lipídio: fator de risco e prevenção do câncer de mama. Revista de Nutrição, Campinas, v. 19, n.1, p. 65-75, jan./fev. 2006.

Recebido em: 15 jul. 2013

Aceito em: 06 set. 2013

Contato: Eloise Werle de Almeida eloisewalmeida@gmail.com

Conexões: revista da Faculdade de Educação Física da UNICAMP, Campinas, v. 11, n. 3, p. 129-146, jul./set. 2013. 